\title{
ON DERIVED AND INTEGRAL BASIC SETS OF POLYNOMIALS
}

\author{
RAGY H. MAKAR
}

1. Introduction. The problem of the derived and integral basic sets of polynomials was first treated by R. Makar and M. Mursi [2] where two results were obtained by a simple method. In a recent paper [3] M. N. Mikhail has proved a number of results first by making use of the product set of basic sets of polynomials and then again by applying the method of [2]. It is evidently clear that the self-dependent method of [2] is far simpler. Further, much progress has been made by A. Tantawi [4, chap. V]. He has proved two main results, but again he has made use of the product set. It is more satisfactory and convenient to establish these results by self-dependent proofs of a simple nature. This is done here in Theorems I and IV. Further, a new main result is added here in Theorem II. These three results together with the first result of [2] (stated here in Theorem III), which is also a main result, leave no gap in the study of the derived and integral basic sets of polynomials.

2. The derived sets. The convergence properties of basic sets of polynomials are mainly classified as follows:

(i) effectiveness in a closed circle $|z| \leqq R$;

(ii) effectiveness in an open circle $|z|<R$;

(iii) effectiveness at the origin (for every function);

(iv) effectiveness at the origin for every integral function;

(v) effectiveness (in the whole plane) for every integral function of order $<\rho$;

(vi) effectiveness in a closed circle $|z| \leqq R$ for every integral function of order $<\rho$;

(vii) effectiveness in an open circle $|z|<R$ for every integral function of order $<\rho$;

(viii) effectiveness at the origin for every integral function of order $<\rho$.

Let $\left\{p_{n}(z)\right\} \equiv p_{0}(z), p_{1}(z), p_{2}(z), \cdots$ be a basic set of polynomials. The derived set $\left\{p_{n}^{\prime}(z)\right\} \equiv p_{0}^{\prime}(z), p_{1}^{\prime}(z), p_{2}^{\prime}(z), \cdots$ is basic but with one additional polynomial which is identically zero if one of the elements of the set $\left\{p_{n}(z)\right\}$ is independent of $z[6, p .8]$. The addition of an assigned polynomial to a basic set does not affect its convergence properties, though the representation of a function be-

Received by the editors August 10, 1953. 
comes not unique. For if a function $f(z)$ is represented uniformly in some domain by a series of the form

$$
f(z)=\sum_{n=0}^{\infty} \alpha_{n} p_{n}(z)
$$

and

$$
p(z)=\sum_{n=0}^{N} \beta_{n} p_{n}(z)
$$

is any assigned polynomial, then the addition of a sum of the form $\sum_{n=0}^{N} a_{n} p_{n}(z)+a p(z)$, which is identically zero, to (1), does not affect the uniform convergence of the infinite series in (1) to $f(z)$. Hence there is no loss of generality in studying the representation of $z^{n}$, $n=0,1,2, \cdots$, by the derived set, obtained by differentiating the representation

(3) $\quad z^{n}=\pi_{n 0} p_{0}(z)+\pi_{n 1} p_{1}(z)+\pi_{n 2} p_{2}(z)+\cdots, \quad n=0,1,2, \cdots$.

THEOREM I. If one or another of the convergence properties ii-v, viiviii is possessed by a basic set $\left\{p_{n}(z)\right\}$, it is also possessed by its derived basic set.

Let us write

(4) $\quad F_{n}(R)=\max _{i, j} \max _{|z|=R}\left|\pi_{n i} p_{i}(z)+\pi_{n, i+1} p_{i+1}(z)+\cdots+\pi_{n j} p_{j}(z)\right|$,

(5) $\quad \chi(R)=\limsup _{n \rightarrow \infty}\left\{F_{n}(R)\right\}^{1 / n}$,

(6) $\quad F(R)=\limsup _{n \rightarrow \infty} \frac{\log F_{n}(R)}{n \log n}$.

The convergence properties of any basic set $\left\{p_{n}(z)\right\}$ are decided by the values of $\chi(R)$ and $F(R)$. We associate with the derived set $\left\{p_{n}^{\prime}(z)\right\}$ the corresponding expressions with dashes.

Differentiating (3) we get

$$
n z^{n-1}=\pi_{n 0} p_{0}^{\prime}(z)+\pi_{n 1} p_{1}^{\prime}(z)+\pi_{n 2} p_{2}^{\prime}(z)+\cdots
$$

so that

$$
\begin{aligned}
n F_{n-1}^{\prime}(r) & =\max _{i, j} \max _{|z|=r}\left|\pi_{n i} p_{i}^{\prime}(z)+\pi_{n, i+1} p_{i+1}^{\prime}(z)+\cdots+\pi_{n j} p_{j}^{\prime}(z)\right| \\
& =\max _{|z|=r}\left|\pi_{n h} p_{h}^{\prime}(z)+\pi_{n, h+1} p_{h+1}^{\prime}(z)+\cdots+\pi_{n k} p_{k}^{\prime}(z)\right|
\end{aligned}
$$


But if $p(z)$ is a polynomial, $M(R)=\left.\max \right|_{z}|=R| p(z) \mid$ and $M^{\prime}(r)$ $=\left.\left.\max \right|_{z}\right|_{=r}\left|p^{\prime}(z)\right|$, then

$$
M^{\prime}(r) \leqq M(R) /(R-r) \quad \text { for all } r<R \text { [5, p. 265]. }
$$

Applying (9) to (8) we get

$$
n F_{n-1}^{\prime}(r) \leqq \frac{1}{R-r} \max _{|z|=R}\left|\pi_{n h} p_{h}(z)+\pi_{n, h+1} p_{h+1}(z)+\cdots+\pi_{n k} p_{k}(z)\right|,
$$

so that by (4)

$$
F_{n-1}^{\prime}(r) \leqq F_{n}(R) / n(R-r) .
$$

From (5), (6), and (10) we get

$$
\begin{array}{ll}
\chi^{\prime}(r) \leqq \chi(R) & \text { for all } r<R, \\
F^{\prime}(r) \leqq F(R) & \text { for all } r<R .
\end{array}
$$

Now:

If $\left\{p_{n}(z)\right\}$ is effective in $|z|<R$ then $\chi(r)<R$ for all $r<R[5$, Theorem 29, p. 38]. Given any $r<R$ there is $r_{1}$ such that $r<r_{1}<R$ and then $\chi^{\prime}(r) \leqq \chi\left(r_{1}\right)<R$ for all $r<R$. This proves (ii). Similarly for (vii), since $F(R) \leqq 1 / \rho$ for all $r<R$ [1, Theorem 2, p. 34].

If $\left\{p_{n}(z)\right\}$ is effective at the origin (for every function) then $\chi(0+)=0$ [6, Theorem 30, Corollary]. Making $R \rightarrow 0+$ in (11), $r \rightarrow 0+$ and we get $\chi^{\prime}(0+) \leqq \chi(0+)=0$. This proves (iii). Similarly for (viii), since $F(0+) \leqq 1 / \rho[1$, Theorem 2$]$.

If $\left\{p_{n}(z)\right\}$ is effective at the origin for every integral function, then $\chi(0+)<\infty$ (a corollary of Theorem 22, p. 32; deduction is the same as in the first few lines of the proof of Theorem 15, p. 29). Hence $\chi^{\prime}(0+)<\infty$ and this proves (iv).

Lastly if $\left\{p_{n}(z)\right\}$ is effective (in the whole plane) for every integral function of order $<\rho$, then $F=\lim _{R \rightarrow \infty}\{F(R)\} \leqq 1 / \rho$ [6, Theorem 43, p. 66 and $\S 9$, p. 67]. Making $r \rightarrow \infty$ in (12), $R \rightarrow \infty$ and we get $F^{\prime} \leqq F$ $\leqq 1 / \rho$, which proves $(\mathrm{v})$.

Our new result is concerned with property (vi).

THEOREM II. If a basic set $\left\{p_{n}(z)\right\}$ satisfying the condition

$$
\lim _{n \rightarrow \infty} \frac{\log D_{n}}{n \log n}=0,
$$

where $D_{n}$ is the degree of the polynomial of highest degree in the representation (3), is effective in $|z| \leqq R$ for every integral function of order $<\rho$, then its derived basic set possesses the same property. 
Condition (13) is best possible in the sense that if it is not satisfied the result is not true.

As in (7),

$$
\begin{aligned}
n F_{n-1}^{\prime}(R) & \leqq \max _{i, j} \max _{|z|=R}\left|\pi_{n i} p_{i}^{\prime}(z)+\pi_{n, i+1} p_{i+1}^{\prime}(z)+\cdots+\pi_{n j} p_{j}^{\prime}(z)\right| \\
& \leqq\left(D_{n}+1\right) \max _{\mid} \max _{|z|=R}\left|\pi_{n i} p_{i}^{\prime}(z)\right| \\
& \leqq\left(D_{n}+1\right) \max _{|z|=R}\left|\pi_{n h} p_{h}^{\prime}(z)\right| \\
& \leqq\left(D_{n}+1\right)\left|\pi_{n h}\right| \cdot \sum_{j=1} j\left|p_{h j}\right| R^{j-1} \\
& \leqq\left(D_{n}+1\right)\left|\pi_{n h}\right|\left(\sum_{j=1} j\right) A_{h}(R) / R \\
& \leqq D_{n}\left(D_{n}+1\right)^{2}\left|\pi_{n h}\right| A_{h}(R) / 2 R,
\end{aligned}
$$

so that by (4)

$$
F_{n-1}^{\prime}(R) \leqq D_{n}\left(D_{n}+1\right)^{2} F_{n}(R) / 2 n R .
$$

Hence applying condition (13) we get

$$
F^{\prime}(R) \leqq F(R)
$$

for all $R$.

Our result follows since the necessary and sufficient condition for property (vi) is that $F(R) \leqq 1 / \rho[1$, Theorem 2, p. 34].

The following example illustrates the best possibility of condition (13).

Consider the Cannon ${ }^{1}$ basic set $\left\{p_{n}(z)\right\}$ defined by

$$
\begin{aligned}
& p_{n}(z)=z^{n} \text { when } n \text { is even or zero, } \\
& p_{n}(z)=z^{n}+z^{h(n)} / 2^{n^{n}} \text { when } n \text { is odd, }
\end{aligned}
$$

where $h(n)$ is the nearest even integer to $n^{n}+n \log n$. In this set

$$
z^{n}=p_{n}(z)-p_{h(n)}(z) / 2^{n^{n}}
$$

when $n$ is odd

so that

${ }^{1}$ A Cannon set is such that $N_{n}$, the number of nonzero coefficients $\pi_{n i}$ in (3), satisfies $N_{n}^{1 / n} \rightarrow 1$ as $n \rightarrow \infty$. In such a set the expression $F_{n}(R)$ is replaced by $\omega_{n}(R)$ $=\sum\left|\pi_{n i}\right| A_{i}(R)$, where $A_{i}(R)=\max _{|z|-R}\left|p_{i}(z)\right|$. A basic set satisfying (13) is not necessarily a Cannon set. 


$$
\omega_{n}(R)=R^{n}+2 R^{h(n)} / 2^{n^{n}} .
$$

Hence taking $R=2$

$$
\omega_{n}(2) \simeq 2^{n}+2^{n \log n+1}
$$

so that

$$
\omega(2)=\limsup _{n \rightarrow \infty} \frac{\log \omega_{n}(2)}{n \log n}=\log 2 .
$$

Thus the set $\left\{p_{n}(z)\right\}$ is effective in $|z| \leqq 2$ for every integral function of order $<1 / \log 2[1$, Theorem 2, p. 34].

The derived set is

$$
\begin{array}{lr}
p_{n}^{\prime}(z)=n z^{n-1} & \text { when } n \text { is even and } \geqq 2, \\
p_{n}^{\prime}(z)=n z^{n-1}+h(n) z^{h(n)-1} / 2^{n^{n}} & \text { when } n \text { is odd, }
\end{array}
$$

and we find when $n$ is odd

$$
\omega_{n-1}^{\prime}(R)=R^{n-1}+2 h(n) R^{h(n)-1} / n \cdot 2^{n n}
$$

so that when $R=2$

$$
\omega_{n-1}^{\prime}(2) \simeq 2^{n-1}+h(n) \cdot 2^{n \log n} / n .
$$

Hence

$$
\omega^{\prime}(2)=\limsup _{n \rightarrow \infty} \frac{\log \omega_{n}^{\prime}(2)}{n \log n}=1+\log 2 .
$$

Thus there are integral functions of order $\rho$ such that $1 /(1+\log 2)$ $<\rho<1 / \log 2$ which are not represented by the derived set $\left\{p_{n}^{\prime}(z)\right\}$ in the circle $|z| \leqq 2[1$, Theorem 2].

This is due to the fact that

$$
\limsup _{n \rightarrow \infty} \frac{\log D_{n}}{n \log n}=1>0 .
$$

In order to have a complete list with the final main results on the derived sets of polynomials, I add here the statement of Theorem 1 of [2] (concerning property (i)).

THEOREM III. If a basic set $\left\{p_{n}(z)\right\}$ satisfying the condition

$$
\lim _{n \rightarrow \infty}\left\{D_{n}\right\}^{1 / n}=1
$$


is effective in a circle $|z| \leqq R$, then its derived basic set, also, is effective in $|z| \leqq R$ [2, pp. 48-49].

Condition (14) is best possible in the sense that if it is not satisfied the result is not true $[2$, p. 50$]$.

Such a basic set is necessarily a Cannon set. This result covers Theorem 1 of [3].

3. The integral sets. If $\left\{p_{n}(z)\right\}$ is a basic set of polynomials, then the integral set

$$
\left\{p_{n}(z)\right\} \equiv p_{0}(z), p_{1}(z), p_{2}(z), \cdots \equiv 1, \quad \int_{0}^{z} p_{0}(z) d z, \quad \int_{0}^{z} p_{1}(z) d z, \cdots
$$

is also basic $[4$, p. 8$]$.

ThEOREM IV. If one or another of the properties i-viii is possessed by a basic set $\left\{p_{n}(z)\right\}$, it is also possessed by its integral set $\left\{p_{n}(z)\right\}$.

Integrating (3) we get

$$
\frac{z^{n+1}}{n+1}=\pi_{n 0} \bar{p}_{1}(z)+\pi_{n 1} \bar{p}_{2}(z)+\pi_{n 2} \bar{p}_{3}(z)+\cdots .
$$

Therefore

$$
\frac{\bar{F}_{n+1}(R)}{n+1}=\max _{i, j} \max _{|z|=R}\left|\pi_{n i} \bar{p}_{i+1}(z)+\pi_{n, i+1} \bar{p}_{i+2}(z)+\cdots+\pi_{n, j} \bar{p}_{j+1}(z)\right|
$$

$$
=\max _{|z|=R}\left|\pi_{n h} \bar{p}_{h+1}(z)+\pi_{n, h+1} \bar{p}_{h+2}(z)+\cdots+\pi_{n k} \bar{p}_{k+1}(z)\right| .
$$

But if

$$
p(z)=\int_{0}^{z} p(z) d z
$$

then

$$
\bar{M}(R) \leqq R \cdot M(R)
$$

where $M(R)$ and $\bar{M}(R)$ are the maximum moduli of $p(z)$ and $\bar{p}(z)$ on $|z|=R$.

Applying (16) to (15) we get

$$
\frac{\bar{F}_{n+1}(R)}{n+1} \leqq R \cdot \max _{|z|=R}\left|\pi_{n h} p_{h}(z)+\pi_{n, h+1} p_{h+1}(z)+\cdots+\pi_{n k} p_{k}(z)\right|
$$

so that by (4) 


$$
\bar{F}_{n+1}(R) \leqq(n+1) R \cdot F_{n}(R) .
$$

Hence

$$
\bar{\chi}(R) \leqq \chi(R) \quad \text { and } \quad \bar{F}(R) \leqq F(R) \quad \text { for all } R .
$$

This proves the stated result.

A self-dependent proof of this result in the case of Cannon sets only is given in [3, Theorem 5]. I am inclined to believe that there is a mistake in the proof which led to the result. It seems that the author has written $A_{i}(R)$ for $\sum_{j}\left|p_{i j}\right| R^{i}$. In fact in the method used in Theorem 5 [3], the number $D_{n}$ appears in some form $\left(\sum_{j} 1 /(j+1) \simeq \log D_{n}\right)$ and this method cannot be made to lead to the inequalities $\bar{\lambda}(R) \leqq \lambda(R),{ }^{2} \bar{\omega}(R) \leqq \omega(R)$ without imposing a condition on $D_{n}$.

4. Corollary and remark. From Theorems I and IV we deduce the following result.

Let $\left\{p_{n}(z)\right\}$ be a basic set of polynomials such that

$$
p_{n 0}=p_{n 1}=\cdots=p_{n s}=0
$$

for all $n>n_{0}$ and where $s$ is a fixed integer, then if any one of the sets $\left\{p_{n}(z)\right\},\left\{p_{n}^{\prime}(z)\right\},\left\{p_{n}^{\prime \prime}(z)\right\}, \cdots,\left\{p_{n}^{(s)}(z)\right\}$ possesses one or other of the properties ii- $v$, vii-viii, then this same property is possessed by all these sets.

This follows at once, since in this case each derived set of order $h$ where $0 \leqq h \leqq s$ is itself the integral set of the derived set of order $h+1$, save for a finite number of polynomials which do not affect the convergence properties of a basic set.

We have also similar results, corresponding to properties (i) and (vi), for a basic set satisfying condition (14) or condition (13) respectively.

But if condition (17) is not satisfied this is not true. For we can impose any order of magnitude on the coefficients $p_{n 0}$, e.g. to make the set $\left\{p_{n}(z)\right\}$ have certain "little" convergence properties and then on differentiation these coefficients disappear and the resulting set will have wider convergence properties. In particular the set $p_{0}(z)=1$, $p_{n}(z)=2^{n^{2}}+z^{n}$ does not possess any of the convergence properties i-viii, whilst its derived set is the unit set $\left\{z^{n}\right\}$ which possesses every sort of convergence properties.

Added March 28, 1954. A different approach to Theorems I and

$2 \lambda(R)$ is the expression associated with a Cannon set, corresponding to the expression $\chi(R)$ associated with a general basic set. 
IV, based on topological issues, has been given by W. F. Newns in $\$ 23$ of his distinguished voluminous paper, On the representation of analytic functions by infinite series, Philos. Trans. Roy. Soc. London Ser. A vol. 245 (1953) pp. 429-468.

\section{REFERENCES}

1. M. T. Eweida, On convergence properties of basic series representing integral functions, Proceedings of the Mathematical and Physical Society of Egypt vol. 4 (1950).

2. Ragy H. Makar and M. Mursi, On the representation of functions by basic series of polynomials, Proceedings of the Mathematical and Physical Society of Egypt vol. 3 (1948).

3. M. N. Mikhail, Derived and integral sets of basic sets of polynomials, Proc. Amer. Math. Soc. vol. 4 (1953).

4. A. Tantawi, Thesis for the M.Sc. Degree, Cairo University, 1950, unpublished.

5. E. C. Titchmarch, The theory of functions, Oxford University Press, 2d ed., 1939.

6. J. M. Whittaker, Sur les séries de base de polynomes quelconques, Paris, GauthierVillars, 1949.

IBRAHIM UNIVERSITY 